\title{
L'expert-conférencier : \\ au-delà du rôle traditionnel et implications nouvelles pour la formation des formateurs
}

\author{
Francine BORDUAS*, Robert L. THIVIERCE* *
}

Résumé Contexte : Le contexte de gestion optimale des soins en pays industrialisé ainsi que les connaissances acquises sur "comment les professionnels continuent à apprendre en exerçant", amènent de nouveaux paradigmes dans le champ de la formation médicale continue (FMC). L'interaction, les sessions de discussions de cas cliniques en petits groupes, la rétroaction (feed-back) sont devenus les nouvelles caractéristiques des activités de FMC. Les experts de contenu auxquels on faisait appel le plus souvent pour donner un conférence magistrale interviennent maintenant différemment. Cela requiert de nouvelles habiletés. But: Présenter les nouveaux rôles et nouvelles habiletés requises des experts de contenu dans les nouveaux modèles de FMC. Méthode: Les données nouvelles concernant la façon dont les médecins apprennent tout au long de leur vie professionnelle sont présentées. Les qualités requises par les nouveaux modèles interactifs sont ensuite décrites. Conclusion : L'expert-conférencier peut améliorer les retombées de ses prestations en grand groupe. Il peut aussi et encore plus aider les collègues à ajuster leur pratique clinique en agissant en petits groupes. Les faire discuter sur des cas cliniques, les laisser trouver les solutions, les aider à trouver l'ordre des priorités, interagir et donner un feedback éclairé, telle est la voie de la nouvelle FMC.

Mots clés Formation médicale continue ; experts de contenu ; conférenciers ; apprentissage basé sur des cas cliniques; ateliers de petits groupes ; formation des formateurs.

Summary Context: The context of optimal care management in the industrialised countries, along with the knowledge we have acquired on how professionals continue to learn in the workplace are bringing new paradigms in the field of CME. Maximised interaction, problem-based learning and case-based discussions in small groups, instant feedback have become the new features of the majority of CME sessions. New roles and skills are now being requested from the experts of content who traditionally were called to deliver a lecture at a scientific meeting. Aims : To describe and discuss the new roles and new communication skills expected from experts of content while presenting at scientific and clinical CME activities. Method: An update of our knowledge on how physicians continue to learn during their professional life is presented first. It is followed by a description of the qualities required by the new formats of interaction used in CME sessions. Conclusion: The expert-lecturer can enhance the outcomes of its speech in front of a large group. Better, he can increase the potential of his communications to help physicians to make adjustments in their clinical decision-making by interacting directly during small group sessions. Letting the participants reflect first and then discuss clinical situations, helping them to prioritise solutions, giving them feedback when needed, are all parts of this pathway of new models in CME. The pathway that goes beyond disseminating knowledge, but facilitates and reinforces the integration of this knowledge into the clinical practice of the learners.

Key words Continuing medical education ; content expert ; lecturer ; problem-based learning ; smallgroup workshops, train the trainer sessions.

Pédagogie Médicale 2001; 2 : 37-41.

* Responsable régionale, Formation médicale continue - Association des médecins omnipraticiens de Québec (AMOQ)

** Vice doyen - Formation professionnelle continue - Faculté de Médecine - Université de Montréal

Pour correspondance s'adresser à :

Francine Borduas - Cinique médicale de Neufchatel - 2350 boulevard Bastien - Suite 2 - Québec, Québec, G2B 1 B5

Canada - Courriel : francineborduas@videotron.ca

Robert L Thivierge - Médecine - Direction - Université de Montréal - CP 6128 - Succ. Centre Ville - Montréa - Canada HVC/J \&

Courriel : robert.thivierge@umontreal.ca 


\section{Références et Techniques}

\section{Contexte}

Traditionnellement, « l'expert » est demandé pour donner une conférence lors d'une session de formation médicale continue (FMC). Avant même d'accepter, son premier réflexe est souvent de penser à ses diapositives. Il prévoit produire des diapositives claires, concises, agrémentées de tableaux et d'illustrations choisies. Cette méthode permet de diffuser de l'information et des données nouvelles. Mais, transmettre des connaissances à des apprenants, est-ce le seul but de la formation médicale continue?

Dans le contexte de gestion optimale des soins qui prédomine dans les pays industrialisés, la FMC est en pleine mutation. Elle vise non seulement à augmenter le bagage de connaissances mais aussi à faciliter l'intégration de ces nouvelles connaissances dans la pratique quotidienne des participants cliniciens. Elle veut les aider à modifier leurs décisions cliniques, à réaliser de nouveaux gestes cliniques, à mettre l'emphase sur de nouvelles priorités mises en lumière par des découvertes récentes dans différents secteurs de la santé et ajuster leurs décisions cliniques. Audelà du transfert des nouvelles connaissances, la FMC veut catalyser des changements d'attitudes du médecin qui se répercuteront dans des changements au niveau des soins aux malades et ultimement au niveau des indices de santé publique.

\section{De nouveaux modèles en FMC}

La nouvelle approche en FMC, l'approche interactive, s'inspire de la philosophie des nouveaux moyens de communication. Interactifs, dirigés vers l'apprenant, ils sollicitent une rétroaction immédiate, une comparaison avec les pairs et un processus d'auto-évaluation. C'est la formule de l'atelier interactif.

Selon cette formule, le participant est mis au défi à l'aide de cas cliniques. A partir de situations cliniques auquels il peut référer, le participant-apprenant analyse le problème, échange, discute avec ses pairs à la recherche de solutions en vue d'en arriver à une décision clinique. Il y a interaction directe entre les pairs et rétroaction immédiate par l'expert. Celui-ci agit comme consultant, il est invité à partager son expertise.

Il peut communiquer de nouvelles données (bref exposé théorique, capsules mini-magistrales), rétroagir avec les participants, les aider à établir des priorités, faire du renforcement, dégager des applications pratiques. L'expert contribue ainsi au processus qui rend l'apprenant mieux habilité à gérer le problème soumis.

\section{Le nouveau rôle de l'expert-conférencier}

Selon l'approche interactive, le professeur qu'est l'expertconférencier est appelé à dépasser la simple transmission des connaissances. Pour aider l'apprenant à tirer partie au maximum de son expérience clinique, l'expert-conférencier soulève l'intérêt de l'apprenant en sélectionnant des problèmes cliniques à résoudre, l'incite à trouver une ou des solution(s) appropriée(s) et l'oriente dans cette recherche de solutions. L'expert-conférencier peut servir de modèle quant à la façon de poser le problème, d'y apporter des solutions et d'évaluer l'efficacité de ces solutions.

Expert du contenu et communicateur, il transmettra non seulement son savoir, mais également ce qu'il fait avec ses connaissances, c'est-à-dire son savoir-faire et son savoir-
Le modèle traditionnel

vs

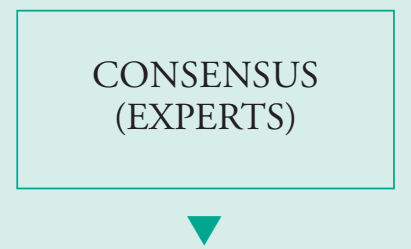

CLINICIENS le modèle interactif

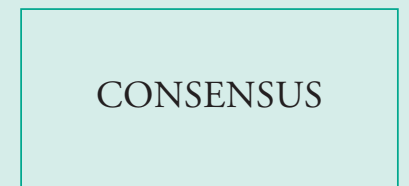

$\triangle$ EXPERTS

CLINICIENS 
être. Le Tableau ci-dessous présente les principaux éléments qui caractérisent le nouveau rôle de l'expert-conférencier.

\section{Préparation de l'expert-conférencier}

Les demandes formulées par les instances organisationnelles en FMC orientent l'expert-conférencier dans sa préparation. Connaissant les besoins de formation et les attentes identifiées auprès de la population cible, l'expert-conférencier pourra mieux centrer ses interventions sur l'apprenant, le participant à l'activité de formation. Les demandes exprimées par les organisateurs en FMC constituent une forme de " contrat pédagogique " pour l'expert-conférencier et les objectifs d'apprentissage traduisent le mandat qui lui est confié. Ils constituent un des éléments-clés dans la préparation de l'expert-conférencier. A partir de ces données, l'expert identifie les messages et concepts importants reliés à chaque objectif . Pour mieux orienter l'apprenant, il priorise les informations c'est-à-dire fait ressortir l'essentiel versus « ce qui est beau à savoir ». Il peut également faire des liens (expliquer le cheminement de sa pensée, expliquer pourquoi une décision marginale est parfois prise considérant les recommandations d'un consensus).

Pour aider l'apprenant à agir avec efficience dès son retour en pratique, l'expert dégage des applications cliniques qui pourront être immédiatement mises en pratique par le médecin participant.

\section{Dirigé vers l'apprenant}

Tout au long de sa préparation et lors de l'activité, l'expert-conférencier fait confiance à l'apprenant. Il tient compte de ses interrogations: "Qu'est-ce que je vais apprendre? " "Qu'est-ce que je vais faire différemment ?", "Qu'est-ce que je vais changer ?"

L'expert considère les attentes, le style d'apprentisage et la façon de faire de l'apprenant qui aime découvrir les connaissances à acquérir, partager ses connaissances et habiletés avec ses pairs, échanger des idées et des expériences, convaincre.

Il considère non seulement le changement dans les connaissances mais aussi le changement au niveau des attitudes et des comportements : ce que je SAIS, ce que je FAIS, ce que je SUIS. Il cherche à développer une pensée critique chez le participant en l'incitant à se poser les bonnes questions : "Qu'est-ce qui arriverait si ? " "Quelle est la force ou la faiblesse de... ?" "Quelle importance cette notion a-t-elle dans ma pratique et pourquoi ?"

Il part du contenu, de concepts fondamentaux et priorise les éléments essentiels. Il considère les véhicules pour transmettre ces concepts ainsi que la façon optimale de les utiliser.

\section{L'expert un communicateur qui transmet}

\begin{tabular}{|l|l|l|}
\hline $\begin{array}{l}\text { Savoir } \\
\text { Transmet l'information }\end{array}$ & $\begin{array}{l}\text { Savoir-faire } \\
\text { Rétroagit avec l'apprenant }\end{array}$ & $\begin{array}{l}\text { Savoir-être } \\
\text { Transmet les messages } \\
\text { de façon non verbale }\end{array}$ \\
Transmet une façon de penser, \\
$\begin{array}{l}\text { Fommunique } \\
\text { les nouvelles données }\end{array}$ & $\begin{array}{l}\text { Fait des liens, facilite } \\
\text { répond aux questions } \\
\text { "ce qu'il est » }\end{array}$ & $\begin{array}{l}\text { Précise l'état actuel des connais- } \\
\text { sances et dégage des applications } \\
\text { pratiques }\end{array}$ \\
$\begin{array}{l}\text { Rappelle les messages } \\
\text { et les concepts importants }\end{array}$ & $\begin{array}{l}\text { Témoigne par son attitude } \\
\text { Corrige les performances jugées }\end{array}$ & \\
\hline
\end{tabular}




\section{Références et Techniques}

\section{Les outils de l'expert-conférencier L'outil-premier de l'expert-conférencier : CE QU'IL EST.}

La façon dont les données sont présentées prime sur les mots et le matériel utilisé qui devrait servir de support à une présentation vivante, dirigée vers l'apprenant. Limpact d'une présentation s'évalue de la façon suivante : $7 \%$ par les mots utilisés, $38 \%$ par la voix et $55 \%$ par le non-verbal. Ainsi, $93 \%$ de l'impression vient de l'apparence et du son d'où l'importance du contact visuel, des gestes, du sourire, d'une voix pausée avec tonalité variée. Une attitude de confiance, d'aisance et d'animation constitue une base solide pour le communicateur.

\section{Les messages-clés}

Identifiés à partir des objectifs, les messages-clés s'expriment verbalement, visuellement et mimiquement. Ils devraient apparaître périodiquement au cours de l'activité de formation et ressortir de façon claire et concise dans une présentation visuelle.

Limiter le nombre de messages importants à 3 ou 4 messages bien ciblés, bien élaborés, favorise la rétention chez l'apprenant.

\section{Les questions}

Les questions constituent des outils prolifiques pour générer l'interaction. La période de questions peut être une excellente occasion pour renforcer les messages-clés.

Dans sa préparation, le conférencier-expert prévoit les questions qui pourront lui être posées, anticipe les questions négatives, prépare des liens avec des renseigments spécifiques et positifs.

Le conférencier-expert se prépare à poser lui-même des questions. Il peut utiliser les questions " POURQUOI ? " pour soulever l'intérêt, capter l'attention, stimuler la discussion, encourager les échanges, guider la réflexion ou évaluer la compréhension.

Répondre aux questions de l'apprenant permet de faire du renforcement, corriger les performances jugées inadéquates et préciser l'état actuel des connaissances.

\section{Les documents photographies}

Le matériel utilisé, qu'il soit sous forme de transparents ou diapositives couleur, sera d'autant plus efficace si les données sont présentées de façon claire, concise et harmonieuse. Parmi toutes les qualités exigées d'un document photographie, la lisibilité est la plus importante.

Les textes courts présentent plusieurs avantages : ils facili- tent la compréhension, clarifient le discours, renforcent le message verbal, retiennent davantage l'attention de l'auditoire, stimulent l'intérêt et permettent au présentateur de s'éloigner de son schéma avec l'assurance que le spectateur pourra toujours s'y référer.

Lors de la préparation des diapositives, la règle de 7 prévaut : 7 lignes de texte à la verticale, 7 mots à l'horizontale. Il peut être avantageux de préparer les textes de sorte que leur grand axe soit horizontal sur l'écran (format paysage). Différents logiciels peuvent assister l'expert-conférencier dans la conception et la préparation de son matériel photographie, diapositives ou transparents. Mais au-delà de toute la technique et des effets visuels, le contenu pédagogique et les messages-clés doivent prédominer.

Voici quelques questions que l'expert pourrait se poser une fois que son texte et ses diapositives sont bien préparées et en ordre :

- Est-ce que chacune des diapositives aide vraiment à atteindre mes objectifs?

- Sont-elles toutes nécessaires?

-Y a-t-il une bonne continuité entre cette diapositive et la précédente?

- Cette diapositive aidera-t-elle l'auditoire à progresser dans ses connaissances?

- L'idée est-elle claire?

- Et cette autre diapositive où j’ai éliminé des éléments sans nuire à la pensée principale, sera-t-elle suffisante avec ses quatre mots?

- L'information que je veux transmettre dans cette dixième diapositive pourra-t-elle être lue et comprise durant les quelques secondes où elle apparaîtra à l'écran ?

- Cette diapositive s'harmonise-t-elle avec mes commentaires verbaux?

\section{Un texte de référence}

Les médecins participant à une activité de formation apprécient recevoir un texte qui pourra servir de référence. Le document peut correspondre au texte de la présentation, aux diapositives présentées, inclure des algorithmes d'investigation ou de traitement. Il peut également renfermer des données complémentaires à la présentation (posologies, statistiques), des éléments à retenir et des références judicieuses pour une consultation ultérieure. Un texte clair, concis avec des messages-clés clairement identifiés constituent un outil précieux pour le participant.

Un texte à compléter en cours de présentation pourrait être remis aux participants. Selon certains, cette approche 
pédagogique favorise la mémorisation des connaissances. Le "document-à-compléter " présente cependant un inconvénient majeur. Le participant qui, lors d'un congrès, doit choisir entre plusieurs sessions, ne peut référer au texte de la présentation à laquelle il n'a pu assister. Le syllabus ou recueil des textes des conférences représente une source d'informations recherchée par les cliniciens ; un texte incomplet peut les priver d'une information pertinente.

\section{En conclusion}

Les nouveaux modèles ne signifient pas la mort de la conférence comme moyen de communication en FMC. La conférence a toujours sa place pour transmettre de nouvelles informations. Inspirée de la formule de l'atelier interactif, une approche interactive, basée sur des études de cas et orientée vers l'apprenant, ajoute une nouvelle dimension à cette méthode pédagogique.

Par l'approche interactive, l'expert-conférencier peut aider le participant à une activité de formation, l'apprenant, à découvrir les connaissances à acquérir, l'orienter dans la recherche de solutions appropriées à des problèmes cliniques et servir de modèle quant à la façon de poser le problème, d'y apporter des solutions et d'évaluer l'efficacité de ces solutions.

Centré sur l'apprenant, attentif à ses besoins et aux demandes des instances organisationnelles en FMC, l'expert-conférencier pragmatique, qui sait communiquer et partager son expertise, est un expert recherché. Ce nouveau rôle, qui va au-delà du rôle traditionnel de conférencier, ouvre sur l'avenir en formation médicale continue avec, demain tout près, l'apprentissage en temps réel.

\section{Lectures complémentaires}

1. Argyris C, Schon D. "Theory in practice: increase profesional effectiveness" San Francisco : JosseyBass, 1992.

2. Davis DA. Evidence for the Effectiveness of $C M E$ JAMA 1992 ; 268: 1111-17.

3. Davis DA, Thomson MA, Oxman AD et all Changing physician performance : a systematic review ot the effect of continuing medical education strategies. JAMA 1995 ; 274 : 700-5.

4. Davis DA, Thomson O'brien $M A$, Freemantle $M$ et al. Impact of formal continuing medical education : do conferences, workshops, rounds and other traditional continuing education activities change physician behavior or health care outcomes? JAMA 1999 ; 282 : 867-74.

5. Davis DA , Fox RD. "The physician as a learner: linking research to practice " Chicago: American Medical Association 1994; 243-80.

6. Delorme P. "La conférence : expertise, communication et showmanship ", L'Organisateur d'EMC, 1990 ; Vol 3 No 1 (éditorial).

7. Frankford DM, Patterson MA, Konrad TR. Transforming practice organizations to foster lifelong learning and committment to medical professionalism. Academic Medecine 2000 ; 75 : 708-17.

8. Negroponte N. Being digital, Boston : First Vintage Bookd,1996.

9. Premi JN. Problem-based, self-directed continuing medical education in a group of practicing family physicians. J Med Educ 1988; 63: 484-6.

10. Schon D. "The reflective practitioner : how profesionals think in action". Cambridge $M A$, Basic Books Inc, 1983.

11. Schon DA. Educating the reflective practitioner: Toward a new design for teaching and learning in the professions. San Francisco Jossey-Bass, 1987.

12. Slotnick HB. How doctors Learn : The Role of Clinical Problems across the Medical School-toPractice Continuum, Academic Medecine 1996 ; $71: 28-34$. 